\section{Developmental changes in craniofacial morphology in subjects with Duchenne muscular dystrophy}

Matsuyuki T, Kitahara T et al. Eur J Orthod 2006; 28: 42-50

In this Japanese population, Duchenne muscular dystrophy (DMD) was associated with developmental variations which might be linked to muscle weakness.

DMD is an X-linked recessive condition in which muscles progressively weaken, and this may play a part in altering craniofacial development. In a Japanese hospital, 35 male subjects had lateral cephalograms taken longitudinally from 10 to 20 yrs of age. No subjects had drugs which could interfere with development. Japanese standards for male development at age 13 and 20 yrs were used as controls.

In young DMD patients, the mean gonial angle was greater, the sagittal length of the cranial base was shorter, and the upper incisors protruded. In adult patients, maxillary alveolus and upper incisors were protruded. Overbite tended to decrease. As a result of changing growth patterns, an initial tendency for clockwise rotation of the mandible was reversed after 16 yrs of age. The authors comment that muscular factors may greatly influence growth and development of the jaws and face.

doi:10.1038/sj.bdj.4813821

\section{ORAL SURGERY; PHARMACOLOGY}

The efficacy of postoperative antibiotic regimens in the open treatment of mandibular fractures: a prospective randomized trial

Miles BA, Potter JK et al. J Oral Maxillofac Surg 2006; 64: 576-582

Antibiotics provided no apparent benefit in this situation.

Pre- and peri-operative antibiotics are of value in the open reduction and internal fixation of mandibular fractures, but it is debatable whether postoperative antibiotics (PA) are necessary. In this study, 291 patients treated in a Texas hospital were alternately (not randomly) allocated to PA. All received pre- and peri-operative antibiotics.

In the PA group, 81 patients completed the study, and in the non-PA group, 100 did so. Follow-up at 1, 3 and 5 wks revealed infection in 22 subjects; 8 of these had PA and 14 did not $(\mathrm{P}=$ 0.4). When infected and non-infected subjects were compared, significantly greater postoperative infection rates were found in Caucasians than Black and Hispanic subjects, and in subjects who abused both alcohol and tobacco.

\section{Chewing ability and quality of life in an 80-year-old population}

Takata Y, Ansai T et al. J Oral Rehabil 2006; 33: 330-334

Chewing ability was associated with overall quality of life (QoL)

This study investigated overall QoL and chewing ability in a group of 1282 80-year-old Japanese subjects, of whom 823 participated. Dental examinations were undertaken and subjects completed questionnaires on QoL and chewing ability. At the time of the study, Japanese versions of oral health-related QoL were not established.

The mean number of teeth was 7.5, and subjects reported they could chew a mean of 11.2 out of 15 specified foods. Some $41 \%$ of subjects were satisfied with their health status, and $84 \%$ were satisfied with daily life. Dissatisfaction with daily life, and with interactions with family or friends, was related to the number of foods which could be chewed. There was no consistent relationship between the remaining number of teeth and QoL.

doi:10.1038/sj.bdj.4813823

BEHAVIOURAL SCIENCE; ORAL HEALTH

\section{Oral health beliefs in adolescence and oral health in young adulthood}

Broadbent JM, Thomson WM et al. J Dent Res 2006; 85: 339-343

In this longitudinal study, early beliefs were related to later oral health.

It has been suggested that changed dental health beliefs should result in changed behaviour. The authors of this New Zealand study aimed to investigate the stability of dental health beliefs in a longitudinal study of a Dunedin birth cohort of 1037 subjects followed up at ages 15, 18 and 26 yrs, when 980 of the cohort were assessed. At these ages, study members were asked to complete a questionnaire about oral-health-related behaviour, and were examined for caries and missing teeth.

At all 3 ages, 742 original cohort members completed the study. Over the 11 years, between 60\% and 100\% held favourable views on 6 dental beliefs regarding fluoride, diet, oral hygiene and visiting the dentist. Subjects holding more favourable beliefs had better self-rated oral health, less bleeding on probing, fewer teeth extracted through caries, less caries, fewer fillings and less plaque. The authors consider they may have underestimated the actual effect of unfavourable dental beliefs. 